http://dx.doi.org/10.11646/phytotaxa.164.1.3

\title{
Allium therinanthum (Amaryllidaceae), a new species from Israel
}

\author{
CRISTIAN BRULLO ${ }^{1}$, SALVATORE BRULLO ${ }^{1 *}$, ORI FRAGMAN-SAPIR ${ }^{2}$, GIANPIETRO GIUSSO DEL \\ GALDO $^{1} \&$ CRISTINA SALMERI ${ }^{3}$ \\ ${ }^{1}$ Dipartimento di Scienze Biologiche, Geologiche e Ambientali, Università di Catania, Via A. Longo 19, I- 95125 Catania, Italy, \\ e-mail: salvo.brullo@gmail.com \\ ${ }^{2}$ Jerusalem Botanical Gardens, Hebrew University, 9190401, Jerusalem, Israel \\ ${ }^{3}$ Dipartimento di Scienze e Tecnologie Biologiche, Chimiche e Farmaceutiche, Università degli Studi di Palermo, Via Archirafi 38, I- \\ 90123 Palermo, Italy \\ "Author for correspondence
}

\begin{abstract}
Allium therinanthum, a new species of $A$. sect. Codonoprasum, is described and illustrated from southern Mt. Hermon (Israel). It is a late-flowering diploid species $(2 \mathrm{n}=16)$, growing on calcareous substrates of the mountain belt. It is a narrowly distributed geophyte, showing morphological relationships mainly with A. tardiflorum, a typical autumnal species also occurring in Israel within the pinewoods of Mt. Carmel. The morphology, karyology, leaf anatomy, ecology, conservation status and taxonomical relations are examined for both species. A taxonomic comparison with the most allied late flowering species of the sect. Codonoprasum is provided.
\end{abstract}

Key words: Alliaceae, Allium sect. Codonoprasum, karyology, late flowering, leaf anatomy, phenetic tree, taxonomy

\section{Introduction}

According to literature (Kollmann 1971, 1973, 1985, 1986, Kollmann \& Stearn 1975, Kollmann \& Shmida 1977, Shmida \& Kollmann 1977, Kollmann et al. 1990, Brullo et al. 1991, 1996, 2008b, Feinbrun-Dothan \& Danin 1998, Fragman-Sapir \& Fritsch 2012), the genus Allium Linnaeus (1753: 294) is represented in Israel by several species (ca. 45), many of which endemic to this territory. Within this genus, particularly interesting are those taxa belonging to the sect. Codonoprasum Rchb. in Mössler (1827: 538), having in the Middle East one of the main centres of differentiation. The most significant species of this section occurring in Israel that are worthy to be mentioned are: Allium tardiflorum Kollmann \& Shmida in Kollmann et al. (1990: 24), A. galileum Brullo et al. (2008: 250), A. daninianum Brullo et al. (1996: 239), A. pseudostamineum Kollmann \& Shmida (1977: 138), A. albotunicatum Schwarz (1934: 73), A. hermoneum (Kollmann \& Shmida 1977: 141) Brullo et al. (2007: 330), A. feinbergii Oppenh. in Oppenheimer \& Evenari (1940: 185), A. sindjarense Boiss. \& Hausskn. ex Regel (1875: 121), and A. rupicola Boiss. ex Mouterde (1966: 273), which are all restricted to Israel or even to some neighboring country (e.g. Lebanon, Syria and Jordan).Other species of this section are widespread in the Mediterranean area or in the Saharo-Arabian region, such as A. pallens Linnaeus (1762: 427), A. dentiferum Webb \& Berthelot (1848: 345), A. desertorum Forsskål (1775: 72), and A. decaisnei Presl (1845: 544).

In the framework of taxonomical investigations on the genus Allium carried out in Israel, an unusual population flowering in summer (July to August) has been found in the western slope of Mt. Hermon (northern Israel). For the habit, bivalve persistent spathe, fastigiate inflorescence, campanulate flowers, and inconspicuous nectaries, these plants clearly fall within the sect. Codonoprasum of the subgen. Allium. Many taxonomical studies have been focused on this section over the last decades, whose main outcomes were the assessment of some critical taxon or even the description of several new species (Bogdanović et al. 2008, 2009, 2011, Brullo et al. 2001, 2003a, 2003b, 2004, 2007, 2008b, 2009, 2010, Tzanoudakis 2000, Tzanoudakis \& Tan 2000, Biel et al. 2006, Peruzzi 2007, Tzanoudakis et al. 2008, Özhatay et al. 2010, Trigas et al. 2010, Koçyiğit \& Özhatay 2012). Among 
From the morphological point of view, Allium therinanthum is well differentiated from the above-mentioned species especially for a series of character-state combinations that allow a clear separation of all of them. In particular, A. therinanthum, for some features regarding the habit, inflorescence and flowers, shows closer relationships with $A$. tardiflorum, a punctiform endemism occurring in Israel, too (Figs. 2D, 5). However, $A$. tardiflorum is characterized by larger bulbs, with outer coats membranaceous and purplish-violet, leaves more numerous and longer, sub-cylindrical, without ribs, shorter than stem, spathe valves unilateral, almost completely fused, the shorter one 3-nerved, tepals yellow-green tinged with purple, longer and markedly unequal, anthers longer, white-yellow, ovate and apiculate, occurrence of interstaminal appendices, ovary shorter, ellipsoid, minutely papillose above and capsule subglobose. Besides, A. tardiflorum behaves as a typical autumnal flowering species (late September-early November), and it grows at a lower elevation (ca. 400-500 m a.s.1.) in pine-woods dominated by Pinus halepensis Miller (1768: without pagination). From the karyological analysis, this species revealed a somatic chromosome number of $2 \mathrm{n}=2 \mathrm{x}=16$ in all examined samples from the type locality (Fig. 6A). This is the first karyological report for A. tardiflorum. Although both taxa share the same chromosome set, the karyotype of $A$. tardiflorum is very different from that one of $A$. therinanthum given that it is characterized by two subterminal chromosome pairs provided with long linear satellites on the short arms, while the remaining chromosomes are more or less metacentric, with an arm ratio ranging from 1.04 to 1.2 (Fig. 6B). The chromosome formula of $A$. tardiflorum can summarised as $2 \mathrm{n}=2 \mathrm{x}=16: 12 \mathrm{~m}+4 \mathrm{st}^{\text {sat }}$. In addition, chromosome size in $A$. tardiflorum is smaller than in A. therinanthum: chromosomes range from $12.22 \pm 1.9 \mu \mathrm{m}$ to 7.20 to $\pm 1.7 \mu \mathrm{m}$. The leaf anatomy also shows relevant differences, because the leaf cross section of $A$. tardiflorum (Fig. $3 \mathrm{~B}$ ) has a subcircular to elliptical and smooth outline, an epidermis covered by a thicker cuticle, with regular cells and more stomata, less (max. 20) and often larger vascular bundles. Besides, $A$. therinanthum can be confused with $A$. galileum especially for its habit; the latter is a species rather common in Israel, usually occurring at lower elevation (200-1000 m a.s.1.). According to Brullo et al. (2008b), A. galileum (Fig. 2C) differs morphologically from $A$. therinanthum in having bulbs usually smaller (max. $22 \times 18 \mathrm{~mm}$ ), 5-6 leaves, spathe valves completely reflexed, effused-subglobose, pedicels 30-70 mm long, tepals apiculate, greenish-yellow, tinged with brown, stamen filaments purplish, shorter (the inners max. $2.5 \mathrm{~mm}$ long), occurrence of interstaminal teeth, and ovary papillose above, 3.2-3.8 mm long. Other differences chiefly regard the karyotype structure, since A. galileum has 8 metacentric chromosomes and one microsatellited pair. Finally, A. galileum flowers earlier (late April to June), and usually grows in open sunny stands.

\section{References}

Biel, B., Tan, K. \& Tzanoudakis, D. (2006) A new autumn-flowering species of Allium (Liliaceae) from the island of Sifnos (Cyclades, Greece). Willdenowia 36: 367-372. http://dx.doi.org/10.3372/wi.36.36132

Bogdanović, S., Brullo, C., Brullo, S., Giusso del Galdo, G., Musarella, C. M. \& Salmeri, C. (2011) Allium achaium Boiss. (Alliaceae), a critical species of Greek flora. Candollea 66: 57-64.

Bogdanović, S., Brullo, S., Giusso del Galdo, G. \& Salmeri, C. (2009) A new autumn-flowering species of Allium (Alliaceae) from Croatia. Folia Geobotanica 44: 83-93. http://dx.doi.org/10.1007/s12224-009-9032-2

Bogdanović, S., Brullo, S., Mitić, B. \& Salmeri, C. (2008) A new species of Allium (Alliaceae) from Dalmatia, Croatia. Botanical Journal of the Linnean Society 158: 106-114. http://dx.doi.org/10.1111/j.1095-8339.2008.00790.x

Boissier, E. (1853) Diagnoses plantarum orientalium novarum, ser. 1, 12. Typis Henrici Wolfrath, Neacomi, 112 pp.

Brullo C., Brullo S., Giusso del Galdo G. \& Salmeri C. (2010) Allium makrianum (Alliaceae), a new autumnal species from Greece. Phyton (Horn) 49: 267-278.

Brullo, F. (2002) CromoLab $\odot$. Version 1.1. Dipartimento di Botanica, Università degli Studi di Catania.

Brullo, S., Giusso del Galdo, G. \& Terrasi, M. C. (2008a) Allium aeginiense Brullo, Giusso \& Terrasi (Alliaceae), a new species from Greece. Candollea 63: 197-203.

Brullo, S., Guglielmo, A., Pavone, P. \& Salmeri, C. (2001) Cytotaxonomical notes on some rare endemic species of Allium (Alliaceae) from Greece. Caryologia 54 (1):37-57. http://dx.doi.org/10.1080/00087114.2001.10589212

Brullo, S., Guglielmo, A., Pavone, P. \& Salmeri, C. (2003a) Considerazioni citotassonomiche e filogenetiche su alcune specie a fioritura autunnale di Allium sez. Codonoprasum dell'area mediterranea. Atti $98^{\circ}$ Congresso della Società Botanica Italiana: 15-16. Catania. 
Brullo, S., Guglielmo, A., Pavone, P. \& Salmeri, C. (2007) Cytotaxonomic considerations on Allium stamineum Boiss. group (Alliaceae). Bocconea 21: 325-343.

Brullo, S., Guglielmo, A., Pavone, P. \& Salmeri, C. (2008b) Taxonomic study on Allium dentiferum Webb \& Berthel. (Alliaceae) and its taxonomic relations with allied species from the Mediterranean. Taxon 57: 243-253.

Brullo, S., Guglielmo, A., Pavone, P., Salmeri, C. \& Terrasi, M.C. (2003b) Three new species of Allium Sect. Codonoprasum from Greece. Plant Biosystems 137: 131-140. http://dx.doi.org/10.1080/11263500312331351391

Brullo, S., Pavone, P. \& Salmeri, C. (1991) Allium kollmannianum, a new species from Israel. F1ora Mediterranea 1: 15-20.

Brullo, S., Pavone, P. \& Salmeri, C. (1996) Allium daninianum (Alliaceae), a new species from Middle East. Willdenowia 26: 237-244.

Brullo, S., Pavone, P. \& Salmeri, C. (1997a) Allium oporinanthum (Alliaceae), a new species from NW Mediterranean area. Anales del Jardin Botanico de Madrid 55: 297-302. http://dx.doi.org/10.3989/ajbm.1997.v55.i2.276

Brullo, S., Pavone, P. \& Salmeri, C. (1997b) Allium anzalonei, eine neue Art für die italienische Flora. Sendtnera 4: 33-39.

Brullo, S., Pavone, P. \& Salmeri, C. (1999) Allium archeotrichon (Alliaceae), a new species from Rhodos (Dodekannisos, Greece). Nordic Journal of Botany 19: 41-46. http://dx.doi.org/10.1111/j.1756-1051.1999.tb01901.x

Brullo, S., Pavone, P., Salmeri, C. \& Scrugli, A. (1994) Cytotaxonomical notes on Allium savii Parl. (Alliaceae), a misappreciated Tyrrhenian element. Candollea 49: 271-279.

Brullo, S., Pavone, P., Salmeri, C. \&. Terrasi, M.C. (2009) Allium garganicum (Alliaceae), a new species from Apulia (SE Italy). Plant Biosystems 143: 78-84. http://dx.doi.org/10.1080/11263500903487765

Brullo, S., Pavone P., Salmeri C. \& Venora G. (2004) Cytotaxonomical investigation on Allium paniculatum ssp. exaltatum (Alliaceae) from Cyprus. Caryologia 57: 274-278. http://dx.doi.org/10.1080/00087114.2004.10589404

Davis, P.H. (1949) On the flora of the nearer East: XIII. Miscellaneous new species and records. Kew Bulletin 4: 97-114. http://dx.doi.org/10.2307/4119046

Feinbrun-Dothan, N. \& Danin, A. (1998) Analytical flora of Eretz-Israel, ed. 2. Publishing House Ltd., Cana, 1008 pp.

Feulgen, R. \& Rossenbach, H. (1924) Mikroscopisch chemischer Nachweiss einer nucleinsaure vom typus der Thymonucleinsaure. Hoppe-Seyler's Zeitschrift für Physiologische Chemie 135: 203-248. http://dx.doi.org/10.1515/bchm2.1924.135.5-6.203

Forsskål, P. (1775) Flora Aegyptiaco-Arabica. Heineck et Faber, Hauniae, 200 pp.

Fragman-Sapir, O. \& Fritsch, R.M. (2012) New species of Allium sect. Melanocrommyum from Eastern Mediterranean. Herbertia 65: 31-49.

Koçyiğit, M. \& Özhatay, N. (2012) Allium maraschicum sp. nov. (Alliaceae) from Turkey. Nordic Journal of Botany 30: 553559. http://dx.doi.org/10.1111/j.1756-1051.2012.01268.x

Kollmann, F. (1971) Allium ampeloprasum L. in Israel (Taxonomy). Israel Journal of Botany 20: 263-272.

Kollmann, F. (1973) Karyology of some species of Allium section Molium in Israel. Israel Journal of Botany 22: 92-112.

Kollmann, F. (1985) The genus Allium in Israel. Rotem 15: 1-136.

Kollmann, F. (1986) Allium. In: Feinbrun-Dothan, D. (ed.) Flora Palaestina 4. The Israel Academy of Sciences and Humanities, Jerusalem, pp. 74-99.

Kollmann, F. \& Shmida, A. (1977) Allium species of Mt. Hermon. I. Taxonomy. Israel Journal of Botany 26: 128-148.

Kollmann, F., Shmida, A. \& Cohen, O. (1990) Allium tardiflorum, a new autumn-flowering species. Herbertia 46 (1): $23-32$.

Kollmann, F. \& Stearn, W.T. (1975) Allium trifoliatum subsp. hirsutum. Israel Journal of Botany 24: 201-204.

IUCN (2010) The IUCN red list of threatened species, version 2010.4. IUCN Red List Unit, Cambridge, U.K. Available from: http://www.iucnredlist.org/. (accessed 10 November 2013).

Levan, A., Freda, K. \& Sandberg, A.A. (1964) Nomenclature for centromeric position on chromosomes. Hereditas 52: 201220.

http://dx.doi.org/10.1111/j.1601-5223.1964.tb01953.x

Linnaeus, C. (1753) Species plantarum, 1. Laurentiis Salvii, Stockholm, Sweden, 560 pp.

Linnaeus, C. (1762) Species Plantarum, ed. 2, vol.1 Laurentii Salvii, Holmie, 784 pp.

Miller, P. (1768) Gardeners Dictionary, ed. 8. Printed from the Author, London

Mössler, J.C. (1827) Gemeinnütziges Handbuch der Gewächskunde, ed. 2, 1. J.F. Hammerich, Altona, 784 pp.

Mouterde, P. (1966) Nouvelle Flore du Liban et de la Syrie 1., Imprimerie Catholique, Beyrouth, 563 pp.

Oppenheimer, H.R. \& Evenari, M. (1940) II. Florula Cisiordanica. Bulletin de la Société Botanique de Genéve 31: $143-423$.

Özhatay, N., Koçyiğit, M. \& Akalın, E. (2010) Allium rumelicum, sect. Codonoprasum, a new species from European Turkey. Phytologia Balcanica 16: 355-359.

Parlatore, F. (1857) Flora italiana 2(2). Le Monnier, Firenze, pp. 221638.

Paszko, B. (2006) A critical review and a new proposal of karyotype asymmetry indices. Plant Systematic and Evolution 258: 39-48. 
http://dx.doi.org/10.1007/s00606-005-0389-2

Peruzzi, L. (2007) Allium garbarii Peruzzi (Alliaceae), a new species endemic to Calabria (S Italy). Candollea 62: 17-25.

Peruzzi, L. \& Eroğlu, H.E. (2013) Karyotype asymmetry: again, how to measure and what to measure? Comparative Cytogenetics 7: 1-9. http://dx.doi.org/10.3897/compcytogen.v7i1.4431

Presl, K. (1845) Botanische Bemerkungen. Abhandlungen der Königlichen Böhmischen Gesellschaft der Wissenschafte, Prague, ser. 5, 3: 431-584.

Rechinger, K.H (1961) Die Flora von Euboea. Botanische Jahrbücher für Systematik. 80: 382-465.

Regel, E. (1875) Alliorum adhuc congnitorum monographia. Trudy Imperatorskago S.-Peterburgskago Botanicheskago Sada 3(2): $1-266$.

Schwarz, O. (1934) Additamentum ad florulam Lydiae, I. Feddes Repertorium Specierum Novarum Regni Vegetabilis 36: 6596.

Shmida, A. \& Kollmann, F. (1977) Allium species of Mt. Hermon. II. Distribution, variation and polyploidy correlated with vertical zonation. Israel Journal of Botany 26: 149-159.

Trigas, P., Iatrou, G. \& Tzanoudakis D. (2010) Allium apergii sp. nov. (Alliaceae, A. sect. Codonoprasum) from Evia island, Greece. Journal of Biological Research-Thessaloniki 14: 225-229.

Tzanoudakis, D. (1983) Karyotypes of ten taxa of Allium section Scorodon from Greece. Caryologia 36: $259-284$. http://dx.doi.org/10.1080/00087114.1983.10797667

Tzanoudakis, D. (2000) Allium aegilicum (Alliaceae), a new autumn flowering species from the island of Antikithira (Greece). Botanika Chronica 13: 81-86.

Tzanoudakis, D. \& Kypriotakis, Z. (1993) Allium platakisii, a new species of the Greek insular flora. Flora Mediterranea 3: 309-314.

Tzanoudakis, D. \& Kypriotakis, Z. (2008) Allium brussalisii (Alliaceae), a new species from Greece. Botanical Journal of Linnean Society 158: 140-146. http://dx.doi.org/10.1111/j.1095-8339.2008.00853.x

Tzanoudakis, D. \& Tan, K. 2000. Allium samothracicum, Tzanoud., Strid \& Kit Tan, a new species from the North Aegean Area, Greece. Portugaliae Acta Biologica 19: 355-360.

Webb, P.B. \& Berthelot, S. (1848) Phytografia canariensis. 3(2), sect. III. Béthune, Paris, pp. 233-479.

Zahariadi, C. (1975) Le sous-genre Codonoprasum (genre Allium L., fam. Alliaceae Agardh, 1858) en Grèce et an Roumanie, IIe partie. Biologia Gallo-Hellenica 6: 27-64. 